\title{
A Study on Prevalence and Antimicrobial Resistance Pattern of Urinary Klebsiella pneumoniae in a Tertiary Care Centre in South India
}

\author{
V. Dillirani ${ }^{1}$ and R. Suresh ${ }^{2 *}$ \\ ${ }^{1}$ Department of Microbiology, Stanley Medical College, Chennai 600001, India \\ ${ }^{2}$ Department of Microbiology, Chengalpattu Medical College, Chengalpattu 603001, India \\ *Corresponding author
}

\begin{tabular}{l} 
K e y w o r d s \\
$\begin{array}{l}\text { Urinary tract infections, } \\
\text { Klebsiella pneumonia, } \\
\text { Antimicrobial resistance, } \\
\text { Extended spectrum beta } \\
\text { lactamases }\end{array}$ \\
\hline Article Info \\
\hline $\begin{array}{l}\text { Accepted: } \\
10 \text { March } 2018 \\
\text { Available Online: } \\
10 \text { April } 2018\end{array}$ \\
\hline
\end{tabular}

\section{Introduction}

Urinary tract infections (UTI) are the commonest bacterial infectious diseases, seeking medical care and antimicrobial therapy worldwide. It is the second most prevalent disease of infectious etiology account for $1-3 \%$ of all consultations in a year (HPA 2009). UTI is defined as presence of the pathogenic microbes in sterile urine, affecting any part of the urinary tract, extending from urethral meatus to the renal cortex. Though highly diverse microorganisms cause UTIs,
Urinary tract infections are the commonest infectious disease, seeking medical consultation and antibiotic therapy worldwide. Klebsiella pneumonia is among the most common uropathogen responsible for recurrent infections and it antimicrobial susceptibility to commonly prescribed drugs vary significantly in different regions. Hence, the objective was to analyze the local etiology and antimicrobial susceptibility pattern of urinary $K$. pneumonia, to optimize the rational empirical therapy. The current study was carried out for a period of one year from March 2016 to February 2017 in a tertiary care centre. Klebsiella pneumonia isolated in this study were $13.8 \%$ (217) of the total isolates and ESBL producing strains were $17.5 \%$ (38), out of which $31(81.5 \%)$ were isolated from hospitalized inpatients. Antibiotic susceptibility pattern revealed that the most effective antimicrobials were Imipenem (91.2\%), Nitrofurantoin (81.6\%) and Amikacin (79.7\%) and beftazidime (70\%) and Ceftriaxone $(63 \%)$. The isolates showed least susceptibility to Ampicillin (19\%), Trimethoprim-Sulphamethoxazole (28.6\%) and Ciprofloxacin (42\%). This study emphasizes the importance of current knowledge of the uropathogens and their susceptibility pattern for the initiation of appropriate empirical therapy and to minimize the evolving resistant strains. 
enteritis, neonatal meningitis and sepsis. Klebsiella is the frequent causative pathogen of nosocomial infections, accounting for 3-7\% of all hospital and healthcare associated bacterial infections (Podschun et al., 1998). In the recent decades appearance of plasmid mediated resistance to extended spectrum beta lactam drugs in hospital settings, is a major cause for therapeutic failure, leading to significant increase in morbidity and mortality. These extended spectrum beta lactamases (ESBLs) were more commonly found in isolates of Klebsiella spp., and less commonly in Escherichia coli (Phillipon et al., 1989). In the last decade advent of carbapenemase producing Klebsiella pneumoniae became a considerable infection control issue.

In developing countries like India UTIs are often managed empirically with wide range of antibacterial agents usually by family physicians, even before the availability of laboratory reports. Worldwide, antimicrobial resistance of urinary $K$. pneumoniae to commonly prescribed drugs vary significantly in different regions (Perez et al., 2007). To optimize the rational empirical therapy, it is vital to know the local etiology and susceptibility patterns of uropathogens. Hence, the present study was conducted to know the antimicrobial susceptibility and resistance pattern of urinary $K$. pneumoniae.

\section{Materials and Methods}

This present retrospective study was conducted in a tertiary care centre for a period of one year from March 2016 to February 2017. Aseptically collected clean catch midstream urine samples in a wide mouth sterile container from both hospitalized inpatients and out patients were processed for bacteriological culture. Urine samples were inoculated using calibrated loops for semiquantitative method on Blood agar and MacConkey agar. The uropathogen isolated were identified to the species level by colony morphology, Gram's staining and conventional biochemical tests.

Klebsiella pneumoniae isolated were tested for antimicrobial susceptibility by Kirby Bauer disc diffusion method, on 5\% Mueller Hinton blood agar, as per CLSI guidelines. All isolates were tested for following antimicrobials - Ampicillin $(10 \mu \mathrm{g})$, Amikacin $(30 \mu \mathrm{g})$, Ciprofloxacin $(5 \mu \mathrm{g})$, Ceftriaxone $(30 \mu \mathrm{g}), \quad$ Ceftazidime $(30 \mu \mathrm{g})$, Imipenem $(30 \mu \mathrm{g}), \quad$ Nitrofurantoin $(300 \mu \mathrm{g}), \quad$ and Trimethoprim-Sulfamethoxazole $(1.25 / 23.75 \mu \mathrm{g})$, as recommended by $\mathrm{CLSI}^{7}$.

Klebsiella pneumonia strains identified as extended spectrum beta lactamases producers (ESBL) producers were confirmed by the double disk synergy test using Ceftazidime and ceftazidime-Clavulanic acid.

\section{Results and Discussion}

In the present study, a total of 1564 organisms were isolated from the 6152 urine samples processed for culture and sensitivity. The most common isolates were Escherichia coli (922) $59 \%$ and followed by Klebsiella pneumonia (217) $13.8 \%$ of the total isolates. Females $(58 \%)$ were more often affected than male $(42 \%)$. Nearly half the population belongs to the age group 21-50 years. Men are affected in their late ages above 50. Female in their reproductive age group. In the present study ESBL producing Klebsiella pneumonia strains were $17.5 \%$ (38), $81.5 \%$ (31) were isolated from hospitalized inpatients and $18.5 \%$ (7) were isolated from out patients (Table 1 and 2).

Antibiotic susceptibility pattern revealed that the most effective antimicrobials were Imipenem (91.2\%), Nitrofurantoin (81.6\%) and Amikacin (79.7\%) and followed by Ceftazidime (70\%) and Ceftriaxone (63\%) (Table 3). 
Table.1 Age and sex distribution of Klebsiella pneumoniae isolates from urine samples

\begin{tabular}{|c|c|c|c|c|c|c|}
\hline \multirow{2}{*}{$\begin{array}{c}\text { Age in } \\
\text { years }\end{array}$} & \multicolumn{2}{|c|}{ Male $(\mathbf{n = 9 1 )}$} & \multicolumn{2}{c|}{ Female $(\mathbf{n = 1 2 6})$} & \multicolumn{2}{c|}{ Total $(\mathbf{n = 2 1 7})$} \\
\hline & $\begin{array}{c}\text { Number of } \\
\text { isolates }\end{array}$ & $\begin{array}{l}\text { Percentage } \\
\%\end{array}$ & $\begin{array}{l}\text { Number of } \\
\text { isolates }\end{array}$ & $\begin{array}{l}\text { Percentage } \\
\%\end{array}$ & $\begin{array}{l}\text { Number of } \\
\text { isolates }\end{array}$ & $\begin{array}{l}\text { Percentage } \\
\%\end{array}$ \\
\hline $\mathbf{1 - 2 0}$ & 9 & 10 & 17 & 13.5 & 26 & 12 \\
\hline $\mathbf{2 1 - 5 0}$ & 38 & 41.7 & 64 & 50.8 & 102 & 47 \\
\hline $\mathbf{5 1 - 8 0}$ & 44 & 48.3 & 45 & 35.7 & 89 & 41 \\
\hline
\end{tabular}

Table.2 Comparison of uropathogens isolated from inpatients and outpatients

\begin{tabular}{|c|c|c|c|c|c|}
\hline \multirow{2}{*}{\multicolumn{2}{|c|}{ Total }} & \multicolumn{2}{|c|}{ Inpatients } & \multicolumn{2}{|c|}{ Outpatients } \\
\hline & & $\begin{array}{l}\text { Number of } \\
\text { organisms }\end{array}$ & $\begin{array}{c}\text { Percentage } \\
\%\end{array}$ & $\begin{array}{l}\text { Number of } \\
\text { organisms }\end{array}$ & $\begin{array}{c}\text { Percentage } \\
\%\end{array}$ \\
\hline \multicolumn{2}{|c|}{ Uropathogens isolated $(n=1564)$} & 976 & 62.4 & 588 & 37.6 \\
\hline \multirow{2}{*}{$\begin{array}{l}\text { Klebsiella } \\
\text { pneumoniae } \\
\text { isolated }\end{array}$} & Total $(n=217)$ & 143 & 66 & 74 & 34 \\
\hline & $\begin{array}{l}\text { ESBL producer } \\
(n=38)\end{array}$ & 31 & 81.5 & 7 & 18.5 \\
\hline
\end{tabular}

Table.3 Antibiotic susceptibility pattern of Klebsiella pneumoniae isolated from urine samples

\begin{tabular}{|l|c|c|}
\hline Antibiotics & Number of Isolates $(\mathbf{n = 2 1 7})$ Sensitive & Percentage \\
\hline Ampicillin & 41 & 19 \\
\hline Amikacin & 173 & 79.7 \\
\hline Ciprofloxacin & 91 & 42 \\
\hline Ceftriaxone & 137 & 63 \\
\hline Ceftazidime & 152 & 70 \\
\hline Imipenem & 198 & 91.2 \\
\hline Nitrofurantoin & 177 & 81.6 \\
\hline Trimethoprim- Sulfamethoxazole & 62 & 28.6 \\
\hline
\end{tabular}

The isolates were highly resistant to Ampicillin, Trimethoprim-Sulphamethoxazole and Ciprofloxacin, the susceptibility to these drugs were $19 \%, 28.6 \%$ and $42 \%$ respectively.

In the recent years, indiscriminate use of broad spectrum antibacterial agents in the treatment of UTIs resulted in significant increase in multiple antibiotic resistant uropathogens. The emergence of multidrug resistant organisms is a major health concern in the community and hospital environment globally (Kumar et al., 2006). Bacterial isolates from patients with UTI showed variable levels of resistance to the tested antibiotics. Especially urinary Klebsiella pneumonia revealed high rates of resistance to commonly prescribed antibiotics. So effective management of the UTIs depends on the causative agent and the selection of optimal susceptible drug.

In the present study the Klebsiella pneumonia was the second most isolated pathogen next to Escherichia coli which was in accordance with the studies conducted in other part of the world (Khameneh et al., 2009; Chin et al., 
2011). In this study UTI occurred more common in females than in males. Among females, frequency of UTI was more among sexually active young women $21-50$ years age group. In males, the frequency of UTI was more in elderly patients (above 50 years), probably due to prostate enlargement and neurogenic bladder. These results were similar with the findings of various Indian studies (Dash et al., 2013; Janakiram et al., 2014; Sood et al., 2010). In our study isolates have shown quite high resistance to commonly prescribed antibacterial agentsAmpicillin, Trimethoprim- Sulphamethoxazole and Ciprofloxacin, the results were in concurrence with previous studies (Metri Basavaraj et al., 2014; Pai et al., 2012). These antibiotics were used more frequently as first line drugs for years in the treatment of UTI in the community has led to it greater resistance. This high level resistance confirms that these agents cannot be currently initiated as first line in empirical therapy of UTI. Amikacin and Nitrofurantoin showed good antimicrobial susceptibility with Klebsiella pneumoniae, therefore should be used preferentially and have gained much importance in the treatment of UTIs.

Second and third generations cephalosporins are widely used in the treatment of infections caused by Klebsiella spp (Romero et al., 2007). Our strains showed moderate antibacterial resistance to the third generation cephalosporins, in contrary to the study conducted by Singh et al., (2003). ESBL producers are most often associated with UTIs (Melzer et al., 2007). The cost of hospitalization and duration of stay and mortality rate are higher with ESBL producing strains of Klebsiella spp. than nonESBL producing Klebsiella spp (Schwaber et al., 2006; Kim et al., 2002). ESBL producing Klebsiella pneumoniae isolated were $17.5 \%$ almost the same results were obtained in the other studies (Romero et al., 2007; El
Bouamri et al., 2015). This study shows that $91.2 \%$ of isolates were susceptible to Imipenem. Carbapenems are the agents of choice for dreadful infections caused by ESBL producers, due to their high susceptibility and high stability to $\beta$ lactamase hydrolysis (Colodner et al., 2004). Antimicrobial agents effective against ESBL producers are very limited, hence Imipenem should be considered as a reserved drug for the treatment of serious infection caused by Klebsiella pneumoniae.

In conclusion, in the present study urinary Klebsiella pneumoniae were resistant to commonly used antimicrobial agents. Our study concludes that, the clinicians are left with very few options in the selection of optimal antimicrobials for empirical treatment of UTI, due to the emergence of higher antimicrobial resistance among uropathogens. This study emphasizes the importance of current knowledge of the uropathogens and their susceptibility pattern for the initiation of appropriate empirical therapy and to minimize the evolving resistant strains. The treating physician should judicially prescribe the drug when needed, based on the antibiogram of the isolated microorganisms and antibiotic policy of the concerned centre.

\section{References}

Chin BS, Kim MS, Han SH, et al., 2011. Risk factors of all-cause in-hospital mortality among Korean elderly bacteremic urinary tract infection (UTI) patients. Archives of Gerontology and Geriatrics 52:e50-e55.

Colodner R, Raz R, et al., 2004. Susceptibility pattern of ESBL producing bacteria isolated from inpatients to five antimicrobial drugs in a community hospital in northern Israel. Int $J$ Antimicrob. Agents 24:409-10.

Dash M, Padhi S, Mohanty I, et al., 2013. Antimicrobial resistance in pathogens causing urinary tract infections in a rural 
community of Odisha, India. $J$ Fam Community Med 20: 20-26.

El Bouamri MC, Arsalane L, et al., 2015. Antimicrobial susceptibility of urinary Klebsiella pneumonia and the emergence of carbepenem-resistant strains: A retrospective study from a university hospital in Morocco, North Africa. African J of Urology 21:36-40.

Health protection agency (HPA) 2009. National standard method. Investigation of urine.

Janakiram B, Sunitha T, et al., 2014. Etiology of urolithiasis from South Indian population: Correlation of recurrence and antibiotic resistance to biofilm production capabilities of uropathogenic microbes. Int J pharm Bio Sci 5(1): 909-9.

Khameneh ZR, Afshar AT, 2009. Antimicrobial susceptibility pattern of urinary tract pathogens. Saudi J Kidney Dis Transpl 20:251-53.

Kim YK, Pai H, 2002. Bloodstream infections by extended spectrum beta - lactamase producing Escherichia coli and Klebsiella pneumonia in children: Epidemiology and clinical outcome. Antimicrob. Agents Chemother. 46(5):1481-91.

Kumar MS, Lakshmi V, Rajagopalan R, 2006. Related Articles, Occurrence of extended spectrum beta-lactamases among Enterobacteriaceae spp. isolated at a tertiary care institute. Indian $J$ Med Microbiol 24(3): 208-11.

Melzer M, Petersen I, 2007. Mortality following bacteremic infection caused by extended spectrum beta - lactamase (ESBL) producing $E$. coli compared to non- ESBL producing E. coli. J, Infect. 55(3):254-59.

Metri Basavaraj C, Jyothi P, 2014. Antimicrobial resistance of Klebsiella pneumonia strains from patients with urinary tract infections in SBMPMC hospital Bijapur, India. Int J pharm Bio Sci 5(3): 376-82.

Pai V, Nair B, 2012. Etiology and sensitivity of uropathogens in outpatients and inpatients with urinary tract infection: Implications on empiric therapy. Ann Trop Med Public Health 5:181-84

Perez F, Endimiani A, Hujer KM, Bonomo RA, 2007. The continuing challenge of ESBLs. Curr Opin Pharmacol 7:459-69

Phillipon A, Labia R, 1989. Extended spectrum beta lactamases. Antimicrobial agents chemother 33:1131-36.

Podschun R, Ullmann U, 1998. Klebsiella spp. as nosocomial pathogens: epidemiology, taxonomy, typing methods and pathogenicity factors. Clin Microbiol Rev 11:589-603.

Romero EDV, Padilla TP, et al., 2007. Prevalence of clinical isolates of Escherichia coli and Klebsiella spp. producing multiple extended-spectrum $\beta$ lactamases. Diagn Microbiol Infect Dis 59:433-37.

Schwaber MJ, Navon-Venezia S, 2006. Clinical and economic impact of bacteremia with extended spectrum beta - lactamase producing Enterobacteriaceae. Antimicrob. Agents Chemother. 50(4):1257-62.

Singh NP, Goyal R, 2003. Changing trends in bacteriology of burns in the burns unit, Delhi, India. Burns 29(2):129-32.

Sood S, Gupta R, 2010. Antibiotic resistance pattern of community acquired uropathogens at a tertiary care hospital in Jaipur, Rajasthan. Indian J Community Med 37:39-44.

\section{How to cite this article:}

Dillirani, V. and Suresh, R. 2018. A Study on Prevalence and Antimicrobial Resistance Pattern of Urinary Klebsiella pneumoniae in a Tertiary Care Centre in South India. Int.J.Curr.Microbiol.App.Sci. 7(04): 969-973. doi: https://doi.org/10.20546/ijcmas.2018.704.103 